\title{
BIT RATE OPTIMIZED TIME-DOMAIN EQUALIZERS FOR DMT SYSTEMS
}

\author{
Chun-Yang Chen, See-May Phoong
}

Dept. of EE and Grad. Inst. of Comm. Engr., National Taiwan Univ., Taiwan, R.O.C.

\begin{abstract}
The discrete multitone (DMT) transceivers have enjoyed great success in high speed data transmission. It is known that when the cyclic prefix is no shorter than the channel impulse response (CIR), the DMT system is ISI free. For channels with very long CIR such as DSL loops, a time-domain equalizer (TEQ) is typically added at the receiver to shorten the effective impulse response. This paper proposes a filterbank approach to the design of TEQ for maximizing the bit rate. Moreover we introduce a structure of DMT system with multiple TEQs. The optimal solution for multiple TEQs is given in closed form and it can serve as a theoretical upper bound for all other TEQs. From the multiple TEQ structure, we propose a DMT system with a pair of complex conjugating TEQs. Simulation examples are given to verify the merit of the proposed TEQ.
\end{abstract}

\section{INTRODUCTION}

Discrete Multitone modulation (DMT) has been successfully employed for high speed data transmission over frequency selective channels such as DSL. Fig. 1 shows a DMT system. In a DMT scheme, the input vector $\mathbf{s}$ consisting of modulation symbols is passed through an $M$-point IDFT matrix. For every block of $M$ data samples, the transmitter adds a cyclic prefix of length $L$. At the receiver, the $L$ samples corresponding to the cyclic prefix are first removed before the DFT operation. It is known that when cyclic prefix is no shorter than the channel impulse response (CIR), we can obtain ISI free by multiplying the DFT output with a set of scalars known as the frequency domain equalizers (FEQ). In a DMT scheme, the longer the CIR is, the longer the cyclic prefix is needed to avoid ISI. For applications such as DSLs where the CIR can be very long, a time-domain equalizer (TEQ) is used to shorten the effective CIR.

In the past, many methods have been proposed for the design of the TEQ [1]-[8]. These methods can be categorized into two types. The first approach is to design the TEQ by optimizing objective functions depending on the TEQ output. In [1] [2] [3], TEQ is designed to shorten the effective CIR or delay spread by maximizing the energy (or weighted) of the effective CIR within a certain window. In [2] [4] [5], the authors design the TEQ so that SNR at the TEQ output is maximized. TEQs designed using these methods are not optimal in the sense that the resulting TEQs do not maximize the bit rate. The second approach optimizes the geometrical mean (GM) of SNRs of all tones [6] [7] [8]. This approach involves highly nonlinear optimization though it is optimal. Suboptimal solution by replacing the GM with arithmetic mean has been given in [8]. However SNR values estimated us-

This work was supported by National Science Council under contract \#NSC91-2219-E-002-047 and Ministry of Education under grant \#89-EFA06-2-4, Taiwan R.O.C. ing formulations in [6] [8] can deviate from the actual values by several decibels.

In this paper, we propose a filterbank (FB) approach to the TEQ design problem. Using this approach, the expressions for the ISI error and noise error at the FEQ output can be obtained. A TEQ minimizing the GM of these error variances can be designed. Moreover the FB approach gives rise to a DMT scheme with multiple TEQs that can be viewed as a generalization of the dual-tone DMT scheme in [9]. The optimal multiple TEQs are given in closed form. Though having a very high implementation cost, DMT scheme with multiple TEQs can serve as a valuable theoretical bound on the performance. Moreover we propose a DMT scheme with a pair of complex conjugating TEQs. Simulations are carried out to demonstrate the usefulness of the proposed scheme.

Boldfaced upper-case and lower-case letters denote matrices and vectors respectively. The symbols ${ }^{*},{ }^{T}$, and ${ }^{H}$ represent respectively complex conjugate, transpose and complex conjugate followed by transpose.

\section{FILTERBANK FORMULATION OF DMT SCHEMES}

Fig. 1 shows a DMT system. In this paper, $M$ denotes the size of the DFT matrix and $L$ represents the cyclic prefix length. The channel is modelled as an LTI real FIR filter $c(n)$ with a real additive WSS noise $\nu(n)$ whose power spectrum is $S_{\nu}\left(e^{j \omega}\right)$. Let $N_{c}$ and $N_{t}$ be respectively the order of the channel $c(n)$ and the time-domain equalizer $t(n)$. Their $z$-domain expressions are

$$
C(z)=\sum_{n=0}^{N_{c}} c(n) z^{-n}, \text { and } T(z)=\sum_{n=0}^{N_{t}} t(n) z^{-n} .
$$

The effective channel becomes $P(z)=C(z) T(z)$. The scalar multipliers $1 / P_{k}$ are known as the frequency-domain equalizers (FEQ), where $P_{k}$ are equal to the product $C\left(e^{j 2 \pi k / M}\right) T\left(e^{j 2 \pi k / M}\right)$.

In the following derivation, we will employ the FB interpretation of DMT transceiver. Using multirate identities, one can verify that operations of cyclic removal, serial-to-parallel conversion and the DFT matrix of Fig. 1 can be redrawn as Fig. 2. The symbol $\downarrow N$ denotes subsampling by a factor of $N$, where $N=M+L$. The receiving filters $H_{k}(z)$ are the DFT filters

$$
H_{k}(z)=\sum_{i=L}^{M+L-1} e^{-j 2 \pi k i / M} z^{i}
$$

In many transmission environments, the channel and noise characteristics vary in different frequency regions. We would like to exploit these characteristics to design a good TEQ. For example, we can use a specific TEQ, say $T_{a}(z)$ for low-frequency tones and another TEQ, say $T_{b}(z)$ for high-frequency tones. By doing so, we are able to optimize $T_{a}(z)$ (or $T_{b}(z)$ ) so that its noise gain at 


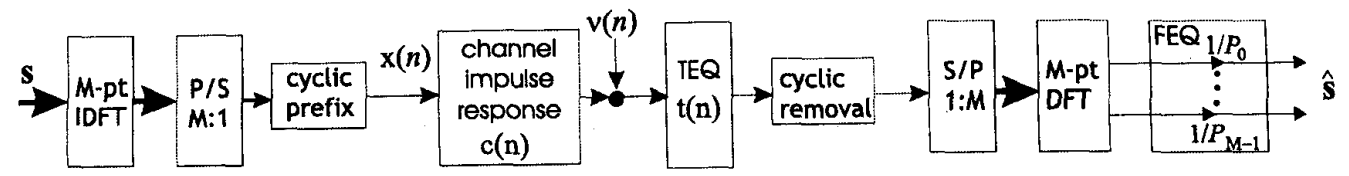

Figure 1: DMT scheme with the time-domain equalizer $t(n)$.

low-frequency (correspondingly high-frequency) region is small. To achieve this flexibility, we introduce a DMT receiver with multiple TEQs as shown in Fig. 3. In this case, the scalars $P_{k}$ are given by

$$
P_{k}=C\left(e^{j 2 \pi k / M}\right) T_{k}\left(e^{j 2 \pi k / M}\right) .
$$

It is not difficult to verify that if the cyclic prefix is no shorter than the impulse responses of shortened channels $C(z) T_{k}(z)$ all $k$, then the DMT scheme with the receiver given in Fig. 3 continues to enjoy the ISI free property. By setting the TEQs $T_{k}(z)=T(z)$ for $k=0, \cdots, M-1$, it is straightforward to verify that Fig. 3 reduces to the conventional case in Fig. 2. In the design of $T_{k}(z)$, we can exploit the extra freedom of the proposed receiver so that the ISI error and noise error are minimized. When only two TEQs are used, then the multiple-TEQ scheme reduces to to the dualtone DMT system in [9]. In Fig. 3, each tone uses a different TEQ and this results in a costly receiver. Though its implementation cost is very high, this multiple-TEQ scheme can serve as a theoretical bound. Later we will see that by carefully designing a pair of complex-conjugating TEQs, one can obtain a very satisfactory performance.

Formulation of ISI Errors and Noise Errors: One of the objectives of TEQ design is that the convolution $c(n) * t_{k}(n)$ will have most of its energy within a specific window of length $L$. Impulse responses outside the window will generate interblock ISI. Define the sequence

$$
d(n)= \begin{cases}0 & \text { for } n_{w}<n \leq n_{w}+L \\ 1 & \text { for } 0 \leq n \leq n_{w} \text { or } n_{w}+L<n \leq N_{c}+N_{t}\end{cases}
$$

where $n_{w}$ is the starting location of the desired window. Then we can describe the ISI term of the $k$ th tone as

$$
p_{i s i, k}(n)=d(n)\left(c(n) * t_{k}(n)\right) .
$$

From Fig. 3, we see that the output error at the $k$ th tone is given by $e_{k}(n)=\left[e_{i s i, k}(n)+e_{\nu, k}(n)\right]_{\downarrow N}$, where

$$
\begin{aligned}
e_{i s i, k}(n) & =h_{k}(n) * p_{i s i, k}(n) * x(n) / P_{k} \\
e_{\nu, k}(n) & =h_{k}(n) * t_{k}(n) * \nu(n) / P_{k} .
\end{aligned}
$$

As the downsampler $[\bullet]_{\downarrow N}$ does not change the variance, we have

$$
\sigma_{e_{k}}^{2}=\sigma_{i s i, k}^{2}+\sigma_{\nu, k}^{2}
$$

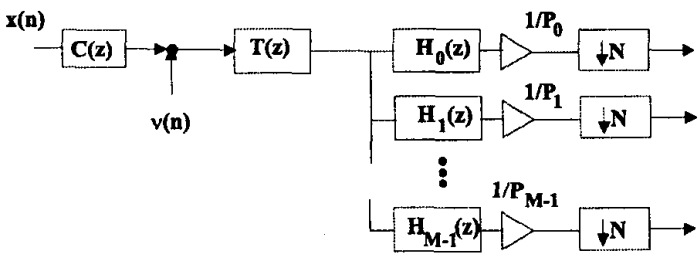

Figure 2: DMT receiver redrawn using the FB structure.

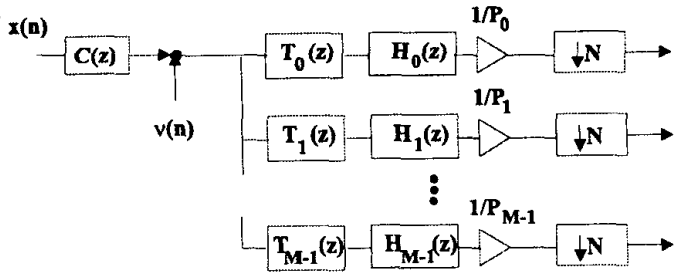

Figure 3: DMT receiver with multiple TEQs $T_{k}(z)$.

where we have assumed that the signal and noise are uncorrelated.

One can express the error variances using a matrix formulation. Define the vectors

$$
\begin{aligned}
\mathbf{t}_{k} & =\left(t_{k}(0) t_{k}(1) \cdots t_{k}\left(N_{t}\right)\right)^{T} \\
\mathbf{w}_{k} & =\left(1 e^{j 2 \pi k / M} \cdots e^{j 2 \pi k N_{t} / M}\right)^{T} .
\end{aligned}
$$

Let $\mathbf{C}$ and $\mathbf{H}_{k}$ be respectively $\left(N_{c}+N_{t}+1\right) \times\left(N_{t}+1\right)$ and $\left(M+N_{c}+N_{t}\right) \times\left(N_{c}+N_{t}+1\right)$ lower triangular Toeplitz matrices whose first columns are given by

$$
\begin{aligned}
& \left(\begin{array}{lllllll}
c(0) & c(1) & \cdots & c\left(N_{c}\right) & 0 & \cdots & 0
\end{array}\right)^{T} \\
& \left(e^{j 2 \pi k(M-1) / M} \cdots e^{j 2 \pi k / M} 10 \cdots 0\right)^{T} \text {. }
\end{aligned}
$$

Let D be an $\left(N_{c}+N_{t}+1\right) \times\left(N_{c}+N_{t}+1\right)$ diagonal matrix with entries $d_{i i}=d(i)$. Using the above definitions, the error variances can be rewritten as ${ }^{1}$ :

$$
\begin{aligned}
\sigma_{i s i, k}^{2} & =\frac{\sigma_{x}^{2} \mathbf{t}_{k}^{H} \mathbf{C}^{H} \mathbf{D}^{H} \mathbf{H}_{k}^{H} \mathbf{H}_{k} \mathbf{D} \mathbf{C}_{k}}{\left|C\left(e^{j 2 \pi k / M}\right)\right|^{2} \mathbf{t}_{k}^{H} \mathbf{w}_{k} \mathbf{w}_{k}^{H} \mathbf{t}_{k}} \\
\sigma_{\nu, k}^{2} & =\frac{\mathbf{t}_{k}^{H} \tilde{\mathbf{H}}_{k}^{H} \mathbf{R}_{\nu} \tilde{\mathbf{H}}_{k} \mathbf{t}_{k}}{\left|C\left(e^{j 2 \pi k / M}\right)\right|^{2} \mathbf{t}_{k}^{H} \mathbf{w}_{k} \mathbf{w}_{k}^{H} \mathbf{t}_{k}},
\end{aligned}
$$

where $\mathbf{R}_{\nu}$ is the $\left(M+N_{t}\right) \times\left(M+N_{t}\right)$ autocorrelation matrix of $\nu(n)$, and $\tilde{\mathbf{H}}_{k}$ is a lower triangular Toeplitz matrix having the same form as $\mathbf{H}_{k}$ but with dimensions of $\left(M+N_{t}\right) \times\left(N_{t}+1\right)$.

\section{OPTIMIZATION OF TEQ}

We have formulated the error variances due to ISI and channel noise. To simplify the notations, we define two $\left(N_{t}+1\right) \times\left(N_{t}+1\right)$ Hermitian matrices:

$$
\mathbf{Q}_{I S I, k}=\frac{\mathcal{E}_{x} \mathbf{C}^{H} \mathbf{D}^{H} \mathbf{H}_{k}^{H} \mathbf{H}_{k} \mathbf{D C}}{\left|C\left(e^{j 2 \pi k / M}\right)\right|^{2}}, \mathbf{Q}_{\nu, k}=\frac{\tilde{\mathbf{H}}_{k}^{H} \mathbf{R}_{\nu} \tilde{\mathbf{H}}_{k}}{\left|C\left(e^{j 2 \pi k / M}\right)\right|^{2}}
$$

\footnotetext{
${ }^{1}$ To simplify the analysis, we assume that $x(n)$ is a white WSS process. This assumption is usually quite accurate when optimal bit and power loading are employed. Numerical simulations show that except for the first tone, all the variances of ISI estimated under this assumption are within 0.5 $\mathrm{dB}$ of the actual values.
} 
Note that the matrix $\mathbf{Q}_{I S I, k}$ is semi positive definite and the matrix $\mathbf{Q}_{\nu, k}$ is positive definite for all $k$. Moreover these matrices satisfy

$$
\mathbf{Q}_{I S I, M-k}=\mathbf{Q}_{I S I, k}^{*}, \mathbf{Q}_{\nu, M-k}=\mathbf{Q}_{\nu, k}^{*},
$$

for $k=1, \cdots, M / 2-1$. For $k=0$ and $k=M / 2$, these matrices are real. In the following, we consider the optimization of the TEQs with different criteria:

A. Single TEQ Minimizing mse at the FEQ output (mmse-f): In this case, $\mathbf{t}_{k}=\mathbf{t}$ for all $k$. The mmse-f TEQ can be obtained by solving the following optimization problem:

$$
\arg \min _{\mathbf{t}} \sum_{k=0}^{M-1} \frac{\mathbf{t}^{H}\left(\mathbf{Q}_{I S I, k}+\mathbf{Q}_{\nu, k}\right) \mathbf{t}}{\mathbf{t}^{H} \mathbf{w}_{k} \mathbf{w}_{k}^{H} \mathbf{t}} .
$$

Note that using the complex conjugate relations in (7) and the fact that $\mathbf{w}_{k}=\mathbf{w}_{M-k}^{*}$, one can verify that the mmse-f TEQ has real coefficients. The above optimization problem is highly nonlinear.

B. Single TEQ Minimizing Geometrical-Mean of $\sigma_{e_{k}}^{2}$ (1-real-opt): It is known [6] [8] that the MMSE-f TEQ is not optimal in terms of bit rate maximization or transmission power minimization. Under bit and power loading, the optimal TEQ is the $t$ that minimizes the following geometrical mean:

$$
\arg \min _{\mathbf{t}} \prod_{k=0}^{M-1} \frac{\mathbf{t}^{H}\left(\mathbf{Q}_{I S I, k}+\mathbf{Q}_{\nu, k}\right) \mathbf{t}}{\mathbf{t}^{H} \mathbf{w}_{k} \mathbf{w}_{k}^{H} \mathbf{t}}
$$

Note that this is the optimal solution for the single TEQ case. Using the same reasoning as above, this optimal TEQ has real coefficients. Such an optimization is also highly nonlinear.

C. Optimal Multiple TEQs (multi-opt): Note that $\mathbf{t}_{k}$ affects only $\sigma_{e_{k}}^{2}$. Therefore when each tone has its own TEQ, the global optimal solution can be obtained by solving

$$
\arg \min _{\mathbf{t}_{k}} \frac{\mathbf{t}_{k}^{H}\left(\mathbf{Q}_{I S I, k}+\mathbf{Q}_{\nu, k}\right) \mathbf{t}_{k}}{\mathbf{t}_{k}^{H} \mathbf{w}_{k} \mathbf{w}_{k}^{H} \mathbf{t}_{k}}
$$

for $k=0, \cdots, M-1$. Note that $\left(\mathbf{Q}_{I S I, k}+\mathbf{Q}_{\nu, k}\right)$ is positive definite. Let $\mathbf{Q}_{k}^{1 / 2}$ be the unique positive definite matrix such that $\mathbf{Q}_{k}^{1 / 2} \mathbf{Q}_{k}^{1 / 2}=\left(\mathbf{Q}_{I S I, k}+\mathbf{Q}_{\nu, k}\right)$. Then by letting $\mathbf{u}_{k}=\mathbf{Q}_{k}^{1 / 2} \mathbf{t}_{k}$, the optimal $\mathbf{t}_{k}$ can be obtained by solving

$$
\arg \max _{\mathbf{u}_{k}} \frac{\mathbf{u}_{k}^{H}\left(\mathbf{Q}_{k}^{-1 / 2}\right) \mathbf{w}_{k} \mathbf{w}_{k}^{H} \mathbf{Q}_{k}^{-1 / 2} \mathbf{u}_{k}}{\mathbf{u}_{k}^{H} \mathbf{u}_{k}} .
$$

As the matrix $\mathbf{Q}_{k}^{-1 / 2} \mathbf{w}_{k} \mathbf{w}_{k}^{H} \mathbf{Q}_{k}^{-1 / 2}$ has rank one, it has only one nonzero eigenvalue and the $\mathbf{u}_{k}$ that maximizes the above function is given by $\mathbf{u}_{k, \text { opt }}=\mathbf{Q}_{k}^{-1 / 2} \mathbf{w}_{k}$. Therefore we have the closed form solution (no nonlinear optimization is needed)

$$
\mathbf{t}_{k, o p t}=\mathbf{Q}_{k}^{-1} \mathbf{w}_{k} .
$$

Note that this is the optimal solution that minimizes the average as well as GM of error variances. The performance of all linear TEQs with the same number of coefficients will be bounded by this solution.

D. Complex-Conjugate Pair of TEQs (2-complex): Though the multi-opt TEQ is globally optimal, its implementation cost is too high. One way to reduce the complexity is to use a small number of TEQs and each TEQ equalizes a number of adjacent tones. The TEQ in each group can be designed separately. From the simulation results on typical CSA loops, we found that it gives a very satisfactory performance if we partition the $M$ tones into 2 groups. Group 1 contains Tones $0, \ldots, M / 2-1$ whereas Group 2 contains Tones $M / 2, \ldots, M-1$. In Group 1, we choose the tone with the highest SNR, say Tone J. The $t_{J, o p t}$ defined in (8) is used as the TEQ for those tones in Group 1. For Group 2, the best tone will be Tone (M-J) due to complex conjugate property and we have $\mathbf{t}_{M-J, o p t}=\mathbf{t}_{J, o p t}^{*}$. As $t_{J, o p t}$ has the closed form solution (8), the design cost of this 2-complex TEQ is very low. Even though there are 2 TEQs, we need only to design and implement one TEQ. The reason is as follows. The outputs of the tones in Group 2 are simply complex conjugates of those in Group 1. At the receiver, we need to implement only those tones in Group 1 and hence only 1 complex TEQ is implemented. Note that this 2-TEQ structure is different from the dual path DMT in [8]. In [8], two receivers, each with a different real TEQ for all tones, are implemented and a tone selector is employed to select outputs with higher SNRs from the two receivers.

\section{SIMULATION EXAMPLES}

The transmission channels considered in the simulation are the 8 typical CSA loops as in [7] [8]. The channel noise consists of a additive white Gaussian noise (AWGN) with $-140 \mathrm{dBm} / \mathrm{Hz}$ and a near-end crosstalk (NEXT) whose power spectral density is

$S_{n e x t}(f)=\gamma k_{N E X T} f^{3 / 2} \times \frac{\left[\sin \left(\pi f / f_{0}\right) \sin \left(\pi f / 2 f_{0}\right)\right]^{2}}{\left[1+\left(f / f_{1,3 d B}\right)^{6}\right]\left(f^{2}+f_{2,3 d B}^{2}\right)}$,

where $f_{0}=1.455 \mathrm{MHz}, f_{1,3 d B}=3 \mathrm{MHz}, f_{2,3 d B}=40 \mathrm{kHz}$ and $k_{N E X T}=2.1581 \times 10^{-9}$. The parameter $\gamma=0.0282(-15.5 \mathrm{~dB})$ represents the adjacent binder effect. The simulation assumes that all the receivers have a perfect estimation of the channel response. The sampling rate $T_{s}$ is $2.208 \mathrm{MHz}$. The DFT size is $M=512$ and the cyclic prefix length is $L=32$. The bits and power are optimally allocated using a water-filling type algorithm. The modulation scheme used is QAM. The order of the TEQ is $N_{t}=4$ (i.e., 5 taps).

We compare the performance of the 4 TEQs in Sec. 3.A-3.D the TEQ that maximizes the signal-to-interference at the TEQ output (maxsir) [1], and the TEQ that minimizes mse at the TEQ output (mmse-t) [2]. In Table 1, we list the maximum achievable bit rate when the transmission power is $\sigma_{x}^{2}=14 \mathrm{dBm}$. The maximum achievable bit rate is given by

$$
\sum_{k=0}^{255} b_{k}=\sum_{k=0}^{255}\left\lfloor\log _{2}\left(1+\frac{\sigma_{x}^{2} / \sigma_{e_{k}}^{2}}{10}\right)\right\rfloor .
$$

For uncoded QAM constellations, the above formula corresponds to a bit error probability of $10^{-7}$. It should be emphasized that the values of $\sigma_{e_{k}}^{2}$ are the true error variances obtained from the actual implementation of the DMT systems.

From Table 1, we see that though having smaller error variances, both mmse TEQs do not necessarily give a better performance than the maxsir TEQ. As expected, the 1-real-opt TEQ outperforms the maxsir, mmse-t and mmse-f TEQs. Despite having a very low design cost, the 2-complex TEQ has an excellent performance and in some cases it outperforms the 1-real-opt TEQ, 


\begin{tabular}{|c|c|c|c|c|c|c|c|c|}
\hline & Loop1 & Loop2 & Loop3 & Loop4 & Loop5 & Loop6 & Loop7 & Loop8 \\
\hline maxsir & 4.93 & 4.14 & 4.83 & 3.98 & 4.42 & 4.49 & 3.93 & 3.30 \\
mmse-t & 4.67 & 5.42 & 4.72 & 4.09 & 4.83 & 4.50 & 3.86 & 3.25 \\
mmse-f & 4.88 & 5.36 & 4.70 & 4.19 & 4.79 & 4.37 & 4.40 & 4.24 \\
1-real-opt & 4.96 & 5.46 & 4.89 & 4.24 & 4.84 & 4.59 & 4.38 & 4.36 \\
2-complex & 4.95 & 5.50 & 4.74 & 4.11 & 4.87 & 4.64 & 4.61 & 4.40 \\
multi-opt & 5.30 & 5.93 & 5.20 & 4.78 & 5.34 & 4.88 & 4.92 & 4.57 \\
\hline
\end{tabular}

Table 1: Maximum achievable bit rate (Mbps). The transmission power is $14 \mathrm{dBm}$.

whose design procedure involves a highly nonlinear optimization problem. The multi-opt TEQ has the best performance.

In [6] [8], the SNR at the $k$ th tone is estimated as follows:

$$
S N R_{k}=\frac{\sigma_{x}^{2}\left|P_{s i g}\left(e^{j 2 \pi k / M}\right)\right|^{2}}{\sigma_{x}^{2}\left|P_{i s i}\left(e^{j 2 \pi k / M}\right)\right|^{2}+S_{\nu}\left(e^{j 2 \pi k / M}\right)\left|T\left(e^{j 2 \pi k / M}\right)\right|^{2}}
$$

where $p_{s i g}(n)=c(n)-p_{i s i}(n)$ represents the impulse response of the signal path. When the noise at the TEQ output is nonflat, this can result in a large error. In Fig. 4, we plot the actual SNRs, SNRs estimated using the FB formulation and SNRs given in (9) versus the subband (or tone) index. The transmission channel in this case is CSA Loop I and the TEQ is maxsir TEQ, whose design is independent of the SNR formula. From the figures, we see that our estimates match nicely with the actual values, whereas SNRs estimated using (9) can deviate from the actual values by as much as $10 \mathrm{~dB}$.

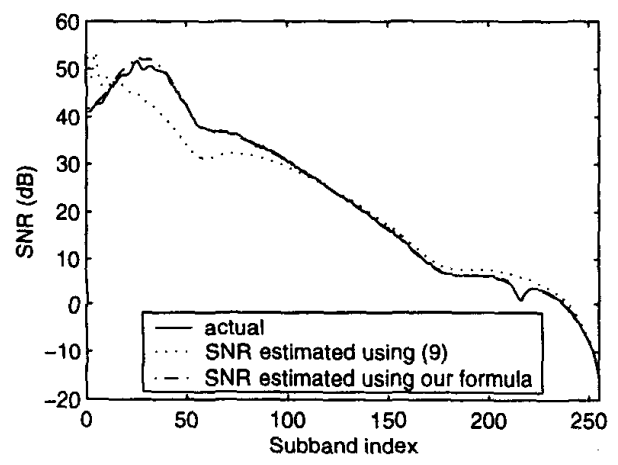

Figure 4: SNR estimated using different formulas.

In Fig. 5, we plot the bit error rate versus transmission power. The transmission channel is CSA Loop 7 and the transmission rate is $6 \mathrm{Mpbs}$. From the figure, we see that multi-opt TEQ has the best performance. The 2-complex TEQ though having a relatively low design and implementation cost is only slightly worse than the multi-opt TEQ. As expected, the 1-real-opt TEQ outperforms the other non optimal 1-real TEQs (maxsir, mmse-t and mmse-f).

\section{CONCLUSIONS}

In this paper, we proposed a FB formulation for the TEQ design problem. Such a formulation gives rises to a DMT structure with multiple TEQs. The optimal solution for the multiple TEQs is given in closed form and its performance can serve as a valuable upper bound. A suboptimal solution with a pair of complex conjugating TEQs is given. Simulations show that the suboptimal so- lution though having a relatively low design and implementation cost is only slightly worse than the multi-opt TEQ.

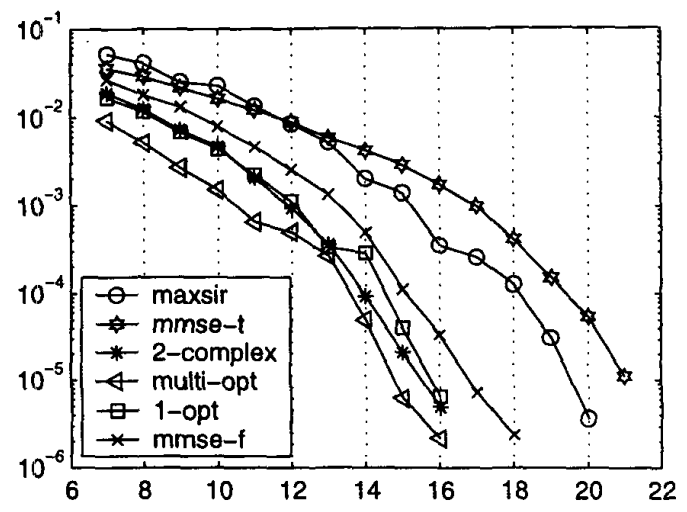

Figure 5: Bit error rate versus transmission power in $\mathrm{dBm}$.

\section{REFERENCES}

[1] P. J. W. Melsa, R. C. Younce, and C. E. Rohrs, "Impulse response shortening for discrete multitone transceivers," IEEE Trans. Commun., Dec. 1996.

[2] I. Djokovic, "MMSE equalizers for DMT systems with and without crosstaik," 31st Asilomar Conf., pp. 545-549, 1997.

[3] R. Schur and J. Speidel, "An efficient equalization method to minimize delay spread in OFDM/DMT systems," Proc. IEEE Int. Conf. Commun., June 2001.

[4] M. Nafie and A. Gatherer, "Time-domain equalizer training for ADSL," Proc. IEEE Int. Conf. Commun., June 1997.

[5] A. Tkacenko and P. P. Vaidyanathan, "Noise optimized eigenfilter design of time-domain equalizers for DMT systems Communications," Proc. IEEE Int. Conf. Commun., 2002.

[6] N. Al-Dhahir and J. M. Cioffi, "Optimum finite-length equalization for multicarrier transceivers," IEEE Trans. Commun., Jan. 1996.

[7] B. Farhang-Bouroujeny and M. Ding, "Design methods for time-domain equalizers in DMT transceivers," IEEE Trans. Commun., Mar. 2001.

[8] G. Arslan, B. L. Evans, and S. Kiaei, "Equalization for discrete multitone transceivers to maximize bit rate," IEEE Trans. Signal Proc, Dec. 2001.

[9] Ming Ding, A. J. Redfern, B..Evans, “dual-path TEQ structure for DMT-ADSL systems," Proc. IEEE Int. Conf. Acoutics. Speech, and Signal Proc, 2002. 